\title{
FBP1 loss contributes to BET inhibitors resistance by undermining c-Myc expression in pancreatic ductal adenocarcinoma
}

Bo Wang ${ }^{1+}$, Ping Fan ${ }^{2+}$, Jingyuan Zhao ${ }^{1}$, Heyu Wu ${ }^{3 *}$, Xin Jin $^{1,2^{*}}$ and Heshui Wu ${ }^{1 *}$

\begin{abstract}
Background: Pancreatic ductal adenocarcinoma (PDAC) is one of the most lethal tumor types worldwide. BET inhibitors display anti-tumor activity in pancreatic cancer, however the cells often develop resistance after a longterm treatment and the underlying molecular basis is not fully understood.

Methods: Drug screening assay in Fructose-1, 6-biphosphatase (FBP1) knockdown or overexpressing pancreatic cancer cells was performed. Tumor cell motility, FBP1 protein and mRNA changes were investigated after BET inhibitors treatment. The interaction between TRIM28 and FBP1 after BET inhibitors treatment was examined by Coimmunoprecipitation (IP) and GST pull-down. The relationship between FBP1 and c-Myc was examined by western blot, RT-qPCR and immunohistochemistry (IHC).

Results: The expression of FBP1 protein increased the sensitivity of pancreatic cancer cells to JQ1. Furthermore, we showed that JQ1 stabilized FBP1 protein level by disrupting the interaction between FBP1 and TRIM28 in pancreatic cancer cells. Moreover, we demonstrated that FBP1 promoted c-Myc degradation through disrupting the ERK-c-Myc axis.

Conclusions: FBP1 modulates the sensitivity of pancreatic cancer cells to BET inhibitors by decreasing the expression of c-Myc. These findings highlight FBP1 could be used as a therapeutic niche for patient-tailored therapies.
\end{abstract}

Keywords: Fructose-1, 6-biphosphatase, C-Myc, Ubiquitination, Degradation, PDAC, BET inhibitors

\section{Background}

Pancreatic ductal adenocarcinoma (PDAC) is one of the most lethal tumor types worldwide and is characterized by a highly invasive and metastatic phenotype [1]. The occurrence and mortality of PDAC remain largely unchanged after decades of studies. The 5-year survival rate is still approximately $5 \%$. Almost all chemotherapeutic agents are ineffective against PDAC [2]. Therefore, a comprehensive understanding of the pathogenesis

\footnotetext{
*Correspondence: why9182008@yahoo.com.cn; jinxinunion@hust.edu.cn; heshuiwu@hust.edu.cn

${ }^{\dagger}$ Bo Wang and Ping Fan contributed equally to this work.

${ }^{3}$ Operating Room, Union Hospital, Tongji Medical College, Huazhong

University of Science and Technology, Wuhan 430022, China

'Department of Pancreatic Surgery, Union Hospital, Tongji Medical College,

Huazhong University of Science and Technology, Wuhan 430022, China

Full list of author information is available at the end of the article
}

of PDAC may unveil novel therapeutic options to improve patient diagnosis.

The bromodomain and extraterminal domain (BET) family, including BRD2, BRD3, BRD4 and BRDT, recognize acetylated histones and regulate gene transcription [3]. BET proteins usually are overexpressed in pancreatic cancer and involved in promoting tumor cell proliferation and metastasis [4]. BET inhibitors have been shown to display anti-tumor activity in KRAS-driven cancers, such as pancreatic ductal adenocarcinoma and non-small cell lung cancer $[4,5]$.

JQ1, one of the most studied selective inhibitors of BET proteins, has been shown the inhibition of pancreatic cancer growth in vitro and in vivo through repression of c-MYC, FOSL and HMGA2 [4, 6, 7]. However, pancreatic cancer cells often develop resistance to BET inhibitors [8]. Interestingly, it is thought that $\mathrm{c}-\mathrm{Myc}$ is

(C) The Author(s). 2018 Open Access This article is distributed under the terms of the Creative Commons Attribution 4.0 International License (http://creativecommons.org/licenses/by/4.0/), which permits unrestricted use, distribution, and 
responsible for this resistance $[9,10]$. The c-Myc protein, an oncogenic transcription factor manipulates at least $15 \%$ of genes associated with cell proliferation, differentiation and metabolism in pancreatic cancer [11]. The KRAS/ERK/c-Myc axis is the major driver of tumorigenesis in pancreatic cancer; therefore, targeting c-Myc is a promising treatment strategy for PDAC $[12,13]$.

PDAC is characterized by an increased aerobic glycolysis [14]. Fructose-1, 6-biphosphatase (FBP1) is a rate -limiting enzyme in gluconeogenesis that converts fructose-1, 6-bisphosphate to fructose-6-phosphate and negatively regulates aerobic glycolysis in pancreatic cancer cells [15]. The reduction of FBP1 expression in pancreatic cancer tissue compared with adjacent normal tissue is associated with a poor prognosis $[16,17]$. Therefore, FBP1 functions as a tumor suppressor in pancreatic cancers. In this study, we hypothesized a novel role of FBP1 in the inhibition of tumor progression in PDAC. We demonstrated that FBP1 increased the sensitivity of pancreatic cancer to JQ1. Moreover, we showed that JQ1 stabilized FBP1 protein level in pancreatic cancer cells, and subsequently promoted c-Myc degradation through the disruption of the ERK-c-Myc axis. Collectively, our result indicated that JQ1 decreased the c-Myc expression partially via stabilizing FBP1 andthe loss of FBP1 contributed to JQ1 resistance in pancreatic cancer.

\section{Methods}

\section{Cell culture}

All pancreatic cancer cell lines including PANC-1 and SW1990 were purchased from the Chinese Academy of Science Cell Bank. These two cell lines were cultured in the Dulbecco's Modified Eagle Medium (DMEM) medium (Invitrogen, USA) supplemented with 10\% fetal bovine serum (FBS) (HyClone, USA). All cell lines were routinely maintained at $37{ }^{\circ} \mathrm{C}, 5 \% \mathrm{CO}_{2}$ incubator.

\section{Plasmids, antibodies and chemicals}

FBP1 wildtype (wt) and FBP1 mutant (G260R) constructs were kindly provided by Dr. Haojie Huang from the Mayo Clinic [11]. The pCMV4a-Flag-c-Myc plasmid was purchased from Addgene. FBP1 nes mutants were generated by adding a nuclear export sequence (LALKLAGLDIGS) to the FBP1 c-terminal using the KOD-Plus- Mutagenesis Kit (Toyobo, Japan). Bacterial expression vectors for GST-TRIM28 recombinant proteins were generated using the pGEX-4 T-1 backbone vector. FBP1 antibody (ab109732)was purchased from Abcam (working dilution 1:1000); beta-Tubulin (2128S) was from Cell Signaling Technology - (working dilution 1:5000); TRIM28 (ab10483) was from Abcam (working dilution 1:3000); c-Myc (5605P) was from Santa Cruz Biotechnology (working dilution 1:1000); BRD2 (ab139690) was from Abcam (working dilution 1:1000);
BRD3 (A302-368A) was from Bethyl Laboratories (working dilution 1:1000); BRD4 (ab128874) was from Abcam (working dilution 1:1000). JQ1, MG132, I CBP112, puromycin and cycloheximide (CHX), were purchased from Sigma-Aldrich (Shanghai, China); MK 2206, Trametinib, GSK126, Ku55933, SB203580 and Palbociclibwere from Selleckchem (Houston, USA). Gemcitabine was obtained from Eli Lilly and Company (Indianapolis, USA). Helenalin was purchased from Cayman Chemical (Ann Arbor, USA).

\section{Western blot of cells and tissue specimens}

The ethics of using human tissue (8 pairs of matched pancreatic cancer/adjacent noncancerous tissues) was approved by the local ethics committee (Tongji Medical College, China), and written informed consent was obtained from patients prior to surgery. The cells or the tissue specimens were lysed with lysis buffer containing $1 \%$ protease and phosphatase inhibitors as described previously [18]. The protein concentration was determined with a protein assay kit (Pierce Biotechnology, USA). Equal amounts of protein for each sample were separated using SDS-PAGE gels and transferred onto PVDF membranes (Pierce Biotechnology, USA). The membranes were subsequently blocked in 5\% not-fat milk for $1 \mathrm{~h}$ at room temperature, followed by incubation with primary antibody overnight at $4{ }^{\circ} \mathrm{C}$. The membranes were then washed with $1 \times \mathrm{TBST}$ and incubated with a secondary antibody for $1 \mathrm{~h}$. Finally, the membranes were treated with ECL detection reagents and exposed to X-ray films.

\section{Real-time RT-PCR}

The total RNA was extracted from the cells using Trizol reagent (Thermo Fisher Scientific, USA). First strand cDNA was synthesized from $1 \mu \mathrm{g}$ of RNA using a cDNA Reverse Transcription kit, and real-time PCR analysis was carried out with a PCR kit according to the manufacturer's protocols. The two kits were purchased from Takara Bio Inc. (Shigo, Japan). All the values were normalized against actin, and the $2-\Delta C t$ method was used to quantify fold change. Primer for RT-qPCR is provided in Additional file 1: Table S1.

\section{Co-immunoprecipitation (co-IP)}

Cells were harvested and lysed by IP buffer $(50 \mathrm{mM}$ Tris- $\mathrm{HCl}, \mathrm{pH}$ 7.4, $150 \mathrm{mM} \mathrm{NaCl}, 1 \%$ Triton X-100, 1\% sodium deoxycholate and $1 \%$ protease inhibitor cocktails) on ice for more than $15 \mathrm{~min}$. Cell lysate was centrifuged for $10 \mathrm{~min}$ at $13,000 \mathrm{rpm}$ at $4{ }^{\circ} \mathrm{C}$, and the supernatant was transferred to a new tube. The supernatant was incubated with primary antibodies and protein A/G agarose beads (Thermo Fisher Scientific, USA) with gentle rocking at $4{ }^{\circ} \mathrm{C}$ overnight. The next day, the 
beads were washed six times with IP buffer on ice, and then subjected to western blotting analysis.

\section{Glutathione S-transferase (GST) pull-down assay}

Cells were lysed with cell lysis buffer $(20 \mathrm{mM}$ Tris- $\mathrm{HCl}$ (pH 7.5), $150 \mathrm{mM} \mathrm{NaCl}, 0.1 \%$ Nonidet P40, $1 \mathrm{mM}$ DTT (dithiothreitol), 10\% glycerol, $1 \mathrm{mM}$ EDTA, $2.5 \mathrm{mM}$ $\mathrm{MgCl} 2$ and $1 \mu \mathrm{g} / \mathrm{ml}$ leupeptin) for $30 \mathrm{~min}$ at $4{ }^{\circ} \mathrm{C}$. GST fusion proteins were immobilized on glutathione-Sepharose beads (GE Healthcare Lifesciences, USA). After washing with lysis buffer, the beads were incubated with cell lysates for $4 \mathrm{~h}$. The beads were then washed four times with binding buffer and re-suspended in sample buffer. The bound proteins were subjected to SDS/PAGE and western blotting analysis.

\section{Tissue microarray and immunohistochemistry (IHC)}

The tissue microarray slides were purchased from Biomax US (lot no. PA1001a). The tissue microarray specimens were immunostained with FBP1 (Abcam, ab109732, working dilution 1:5000) and c-Myc antibodies (Santa Cruz Biotechnology, 5605P working dilution 1:100) as described previously $[17,19]$. Staining intensity was scored in a blinded fashion: $1=$ weak staining at 100x magnification but little or no staining at $40 \times$ magnification; $2=$ medium staining at $40 \times$ magnification; $3=$ strong staining at $40 \times$ magnification. The degree of immunostaining was reviewed and scored by two independent pathologists who were blinded to the clinical details. The scores were determined by the percentage of positive cells multiplied by the staining intensity.

\section{RNA interference}

The lentivirus-based control and gene-specific shRNAs were purchased from Sigma-Aldrich. Lipofectamine 2000 was used to transfect 293 T cells with shRNA plasmids and viral packaging plasmids (pVSV-G and pEXQV). After $24 \mathrm{~h}$ transfection, the medium was replaced with fresh DMEM, containing 10\% FBS and $1 \mathrm{mM}$ of sodium Pyruvate. Next, $48 \mathrm{~h}$ post transfection, the virus culture medium was collected and added to PANC-1 or SW1990 cells supplemented with $12 \mu \mathrm{g} / \mathrm{ml}$ of polybrene. After $24 \mathrm{~h}$ infection, the infected cells were selected with $10 \mu \mathrm{g} / \mathrm{ml}$ of puromycin. The shRNA sequence information is provided in Additional file 1: Table S2.

\section{Cell proliferation assay}

Cell viability was evaluated using the MTS assay according to the manufacturer's instructions (Abcam, USA). Briefly, the pancreatic cancer cells $\left(1 \times 10^{3}\right.$ cells $)$ were seeded in 96-well plates with $100 \mu$ l of culture medium. The cells were treated with serial concentrations of small molecular inhibitors. After $72 \mathrm{~h}$, each well of the cells was added $20 \mu \mathrm{l}$ of MTS reagent (Abcam, USA) and incubated for $1 \mathrm{~h}$ at $37{ }^{\circ} \mathrm{C}$ in standard culture conditions. The absorbance was measured in a microplate reader at $490 \mathrm{~nm}$.

\section{Glucose consumption and lactate production measurement assay}

Pancreatic cancer cells $\left(5 \times 10^{4}\right)$ were seeded in 6-well plates and cultured in DMEM medium without phenol red (Invitrogen, USA). After $24 \mathrm{~h}$ plasmid transfection or $48 \mathrm{~h}$ lentivirus transduction, the spent medium was collected. Glucose concentrations in the spent medium were measured using a glucose (GO) assay kit, according to the manufacturer's instructions (Sigma-Aldrich, USA). Glucose consumption was calculated by subtracting the difference in glucose concentrations between the spent medium and the unused medium. Lactate levels were measured using a lactate assay kit (Eton Bioscience, USA).

\section{Generation of PDAC xenografts in mice}

The BALB/c-nu mice (4-5 weeks of age, 18-20 g) were purchased from Vitalriver (Beijing, China) and randomly divided into four groups ( $n=4$ /group) for subcutaneously inoculation with $5 \times 10^{6}$ of PANC- 1 cells lentivirus infected with shControl, shFBP1, shc-Myc or both shFBP1 plus shc-Myc in the left dorsal flank of mice. The tumors were examined every other day for 21 days; the length and width measurements were obtained with calipers to caculate the tumor volumes by using the eq. $\left(\mathrm{LxW}^{2}\right) / 2$. On day 21 , the animals were euthanized, and the tumors were excised and weighed. All the animal experimental procedures were approved by the Ethics Committee of Tongji Medical College, Huazhong University of Science and Technology.

\section{Statistical analysis}

Statistical analyses were performed with one-sided or two-sided paired Student's t-test for single comparison and one-way ANOVA with a post-hoc test for multiple comparisons. $p$ value $<0.05$ was considered statistically significant. All the values are expressed as the means $\pm \mathrm{SD}$.

\section{Results \\ FBP1 is responsible for modulating the BET inhibitor sensitivity in PDAC}

The $F B P 1$ is a well-known tumor suppressor gene that exhibits low expression or loss of expression in many types of solid tumors [20-22]. Given the importance of the inhibition of cancer progression by FBP1 and the unclear underlying molecular mechanism for this, we performed a drug screening assay in FBP1 knockdown or overexpressing pancreatic cancer cells (PANC-1) and compared the IC50 values of each small molecule with that of the controls (Fig. 1a). We found that the IC50 


\section{a}

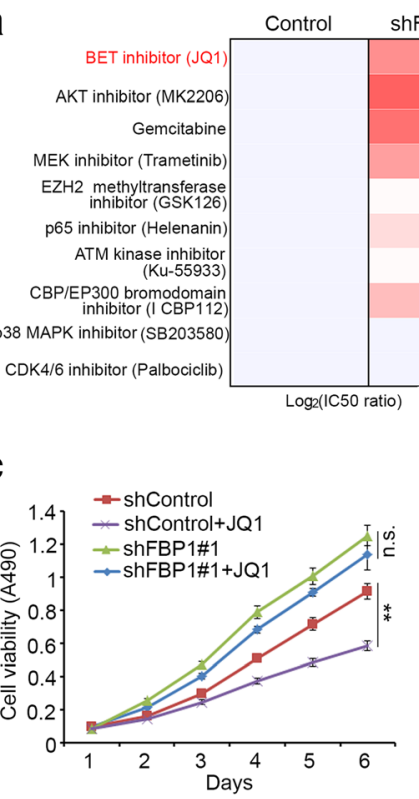

f

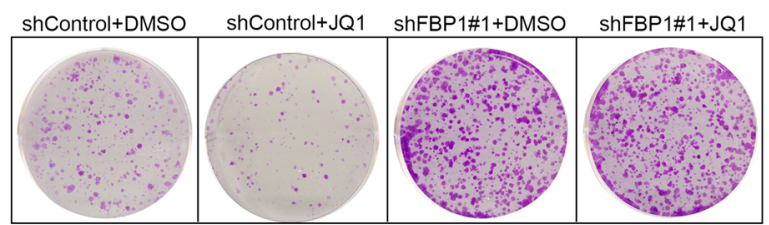

PANC-1
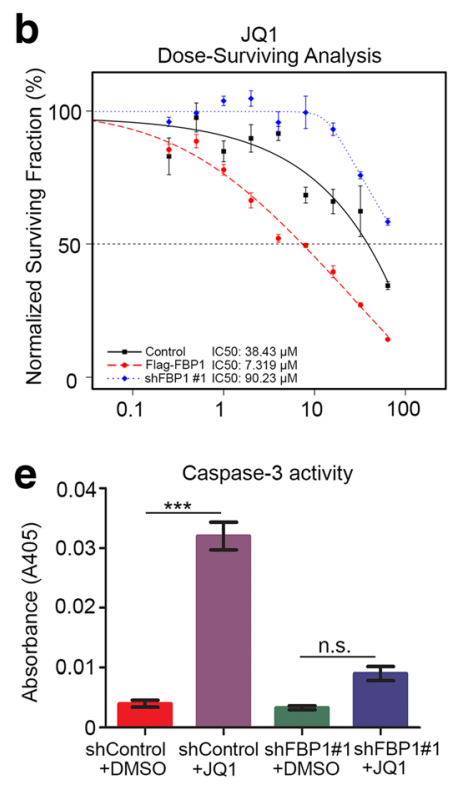

PANC-1

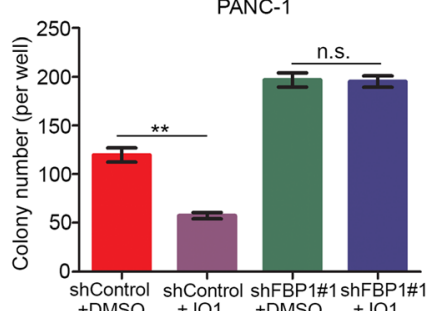

Fig. 1 FBP1 is responsible for modulating the BET inhibitor sensitivity in PDAC. a, PANC-1 cells were infected with lentivirus expressing control, FBP1-specific shRNAs. After $48 \mathrm{~h}$ infection, shControl cells were transfected with pcDNA3.1 or Flag-FBP1 constructs. All cells were treated with different doses of indicated chemicals $24 \mathrm{~h}$ post-transfection. The cell viability was measured by MTS assay. Heat map showing the IC50 ratio ( $\log _{2}($ IC50 ratio)) between shControl versus shControl, knockdown FBP1 versus shcontrol or overexpression FBP1 versus control treated with indicated chemicals. b, PANC-1 cells were infected with lentivirus expressing control, FBP1-specific shRNAs. After $48 \mathrm{~h}$ infection, shControl cells were transfected with pcDNA3.1 or Flag-FBP1 constructs. Cells were treated with different doses of JQ1 24 h post-transfection. The cell viability was measured by MTS assay. Data shown are mean values \pm SD from six replicates. c-f, PANC-1 cells were infected with lentivirus expressing control or FBP1-specific shRNAs. After $72 \mathrm{~h}$ infection, cells were harvested for MTS assay (c), western blotting (d), caspase 3 activity assay (e) and colony formation assay (f). All data are shown as mean values $\pm \mathrm{SD}(n=3)$. n.S., not significant, ${ }^{* *} p<0.01$ comparing to the shControl group

values of JQ1, the most studied BET inhibitor [23], in FBP1 knockdown group was higher than control group (Fig. 1a and b). In contrast, the IC50 value of JQ1 in FBP1 overexpression group was lower than that of the control (Fig. 1a and b). These data suggest that FBP1 is involved in regulating JQ1 sensitivity in pancreatic cancer (Fig. 1b), using gemcitabine as a positive control (Fig. 1a), consistent with previous findings showing that FBP1 loss is responsible for gemcitabine resistance in pancreatic cancer [17]. In order to verify the role of FBP1 in sensitizing PDAC cells to JQ1-induced apoptotic death, PANC-1 cells were treated with JQ1 alone or in combination with FBP1-targeted shRNAs. The knockdown of FBP1 not only increased the pancreatic cancer cells viability (Fig. 1c and f), but also promoted PANC-1 cell resistant to JQ1 drug via decreasing the cleaved PARP expression and caspase-3 activity (Fig. 1c-1f).
Together, our data indicate that FBP1 loss plays a vital role in BET inhibitors resistance in PDAC cells.

\section{JQ1 positively regulates FBP1 protein stability in PDAC}

Given that FBP1 regulates the sensitivity of JQ1 in PDAC cells, the underlying mechanism is unclear. Intriguingly, the data in Fig. 1d indicate that JQ1 increase the expression of FBP1 in PANC-1 cells. Therefore, we use different pancreatic cancer cell lines, PANC-1 and SW1990 to verify this phenomenon. We showed that JQ1 treatment increased FBP1 protein level (Fig. 2a and e) but not the mRNA level (Fig. 2b and f) in dose-and time- dependent manners in both PANC-1 and SW1990 pancreatic cancer cells. Since JQ1 mainly block the transcriptional activity of Bromodomain and Extra-Terminal motif (BET) proteins BRD2, BRD3, BRD4, and BRDT $[24,25]$, we knocked down of BRD2, BRD3 or BRD4 


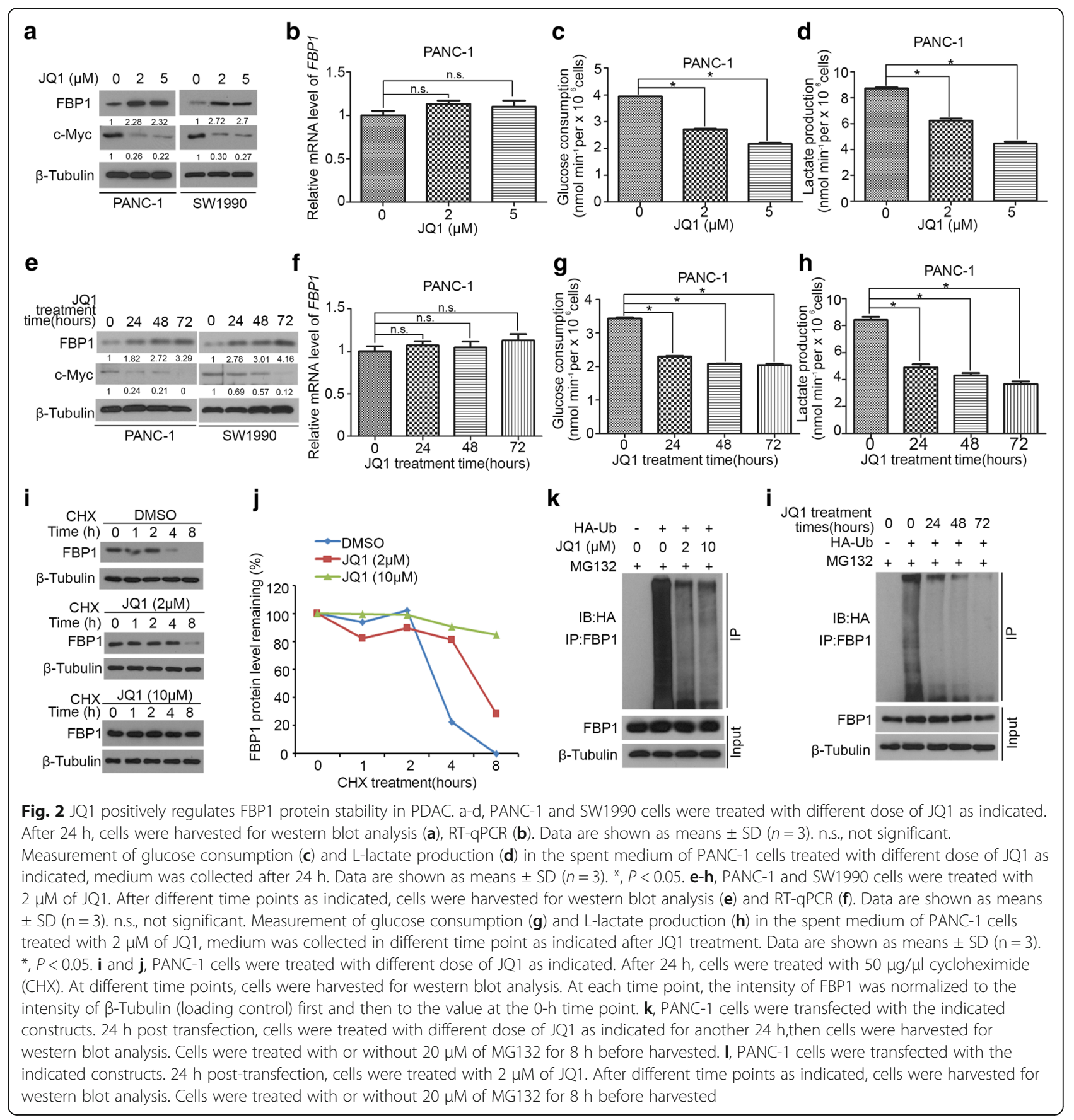

respectively with corresponding specific shRNAs in PANC-1 cells (Additional file 1: Figure S1a, c and 1e) and found that neither knockdown of BRD2, BRD3 nor BRD4 changed the expression of FBP1 in PANC-1 cells (Additional file 1: Figure. S1a-f). Furthermore, due to FBP1 acting as a key enzyme in gluconeogenesis that antagonizes "Warburg effect", we found that JQ1 treatment prevented the glucose consumption and lactate production in PANC-1 cells (Fig. 2c, d, g and h), which was consistent with the alteration of FBP1.
Since JQ1 increased FBP1 protein level which was independent of mRNA level changes, we speculated that JQ1 elevate FBP1 expression through regulating FBP1 post-translational modifications (PTM). Ubiquitination is one of important post-translational modifications described previously [26]. We found that JQ1 prolonged the half-life of FBP1 protein in a dose dependent manner in PANC-1 cells (Fig. 2i and j). Moreover, JQ1 inhibited FBP1polyubiquitination in dose- and time- dependent manners in PANC-1 cells (Fig. $2 \mathrm{k}$ and 1). Collectively, 
these data indicate that JQ1 is capable of inhibiting polyubiquitination and degradation of FBP1 in PDAC.

\section{JQ1 increases FBP1 protein level through disrupting the interaction between FBP1 and TRIM28}

It has been reported previously that TRIM28 functions as an E3 ligase of FBP1 in Hepatocellular carcinoma [27]. Indeed, we confirmed the interaction of endogenous FBP1 and TRIM28 in PDAC cell lines (Fig. 3a) and found that the BROMO domain of TRIM28 mediated the binding with FBP1 (Fig. 3b). Due to JQ1 is one of the BET domain inhibitors, we want to investigate whether BET inhibitors stabilize FBP1 through TRIM28. We found that the interaction between FBP1 and TRIM28 was disrupted when JQ1 was added to the immunoprecipitation reaction (Fig. 3c and d). Moreover, we showed that JQ1 could not cause any obvious effect on FBP1 expression in PDAC cell lines after the knockdown of TRIM28 (Fig. 3e and f). Consistent with the above finding, glucose consumption and lactate

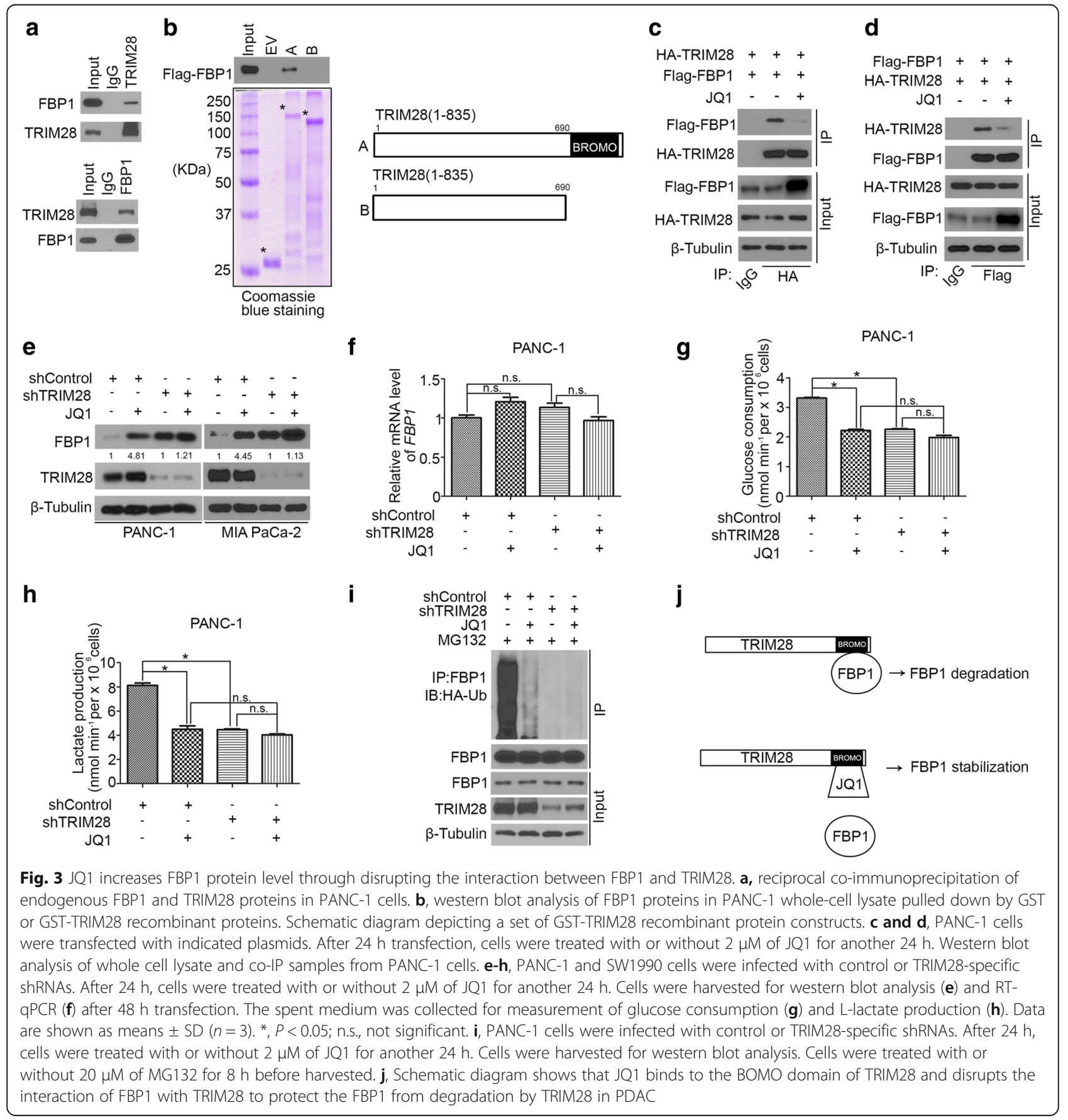


production were also decreased after JQ1 treatment and this process was blocked by the knockdown of TRIM28 (Fig. 3g and h). Furthermore, we found that JQ1 could no longer reduce FBP1 polyubiquitination after TRIM28 knockdown in PDAC (Fig. 3i). Together, these data suggest that BET inhibitors increase FBP1 protein level through disrupting the interaction between FBP1 and TRIM28 (Fig. 3j).

\section{FBP1 negatively regulates c-Myc in PDAC}

It has been reported that $\mathrm{c}-\mathrm{Myc}$ mediates the resistance of pancreatic cancer cells to the BET bromodomain inhibitor JQ1 $[9,10]$, however the underlying mechanism is under investigation. Therefore, we want to determine whether FBP1 participates in modulating the expression of $\mathrm{c}-\mathrm{Myc}$ in pancreatic cancer. Firstly, we found that the knockdown of FBP1 increased c-Myc protein level but not the mRNA level in PANC-1 and SW1990 cells (Fig. 4a and b). Then, the restoration of FBP1 after its knockdown decreased c-Myc protein levels but not the mRNA level in PANC-1 cells (Fig. 4c and d). These finding suggest that FBP1 negatively regulates $\mathrm{c}-\mathrm{Myc}$ protein levels in pancreatic cancer cells.

To investigate the clinical relevance of FBP1 in regulating c-Myc protein levels in pancreatic cancer patients, we assessed both c-Myc and FBP1 protein levels in 8 non-tumor and tumor-paired human pancreatic cancer specimens (Fig. 4e). We found that c-Myc expression was up-regulated in pancreatic cancer tissues compared with adjacent normal pancreatic tissue (Fig. $4 \mathrm{e}$ and $\mathrm{f}$ ). In contrast, the protein level of FBP1 was lower in pancreatic cancer tissues compared to adjacent normal control tissues (Fig. 4e and F). Intriguingly, there was no correlation between $F B P 1$ and $M y c$ at the mRNA level (Fig. $4 \mathrm{~g})$. Meanwhile, we used a tissue microarray of human pancreatic cancer specimens obtained from a cohort of patients ( $n=47$ PDAC tissue specimens) [18] to further examine the correlation between FBP1 and c-Myc at the protein level by IHC (Fig. 4h). Statistical analysis of IHC staining revealed that the c-Myc level was inversely correlated with FBP1 in this cohort (Pearson's product-moment correlation $r=-0.453, p=0.000994$ ) (Fig. 4i). Thus, these data suggest that c-Myc is inversely correlated with FBP1 at the protein level in PDAC patient specimens.

\section{FBP1 promotes c-Myc degradation in pancreatic cancer cells}

It was noteworthy that FBP1 regulated $\mathrm{c}-\mathrm{Myc}$ protein levels but not its mRNA level in pancreatic cancer cells (Fig. 4a-d). Consistent with this finding in cultured cells, our clinical data also demonstrated that the FBP1 protein level was inversely correlated with the $\mathrm{c}-\mathrm{Myc}$ protein level (Fig. 4e), while the FBP1 mRNA level was not overtly correlated with that of $M y c$ (Fig. 4g). Our data suggested that FBP1 regulated c-Myc expression by influencing its post-translational modifications. We systematically investigated whether FBP1 regulate the stability of c-Myc protein in PDAC cells. The overexpression of Flag-FBP1 decreased the protein level of c-Myc in PANC-1 cells, and this effect of FBP1 was completely blocked by the treatment of the proteasome inhibitor MG132 (Fig. 5a and b). Moreover, the knockdown of endogenous FBP1 prolonged the endogenous c-Myc protein half-life in PANC-1 cells (Fig. 5c and d). In agreement with these findings, the knockdown of FBP1 attenuated c-Myc polyubiquitination (Fig. 5e). Therefore, our data indicate that FBP1 promotes the polyubiquitination and degradation of FBP1.

FBP1 is a key enzyme in gluconeogenesis and antagonizes the process of glycolysis [15]. To test whether the enzymatic activity of FBP1 is required for regulating the degradation of c-Myc, we overexpressed the catalytically inactive mutant FBP1 (G260R) [15], and observed a similar inhibitory effect on c-Myc protein level compared with the FBP1 WT in PANC-1 cells (Fig. 5f-5j). These data suggest that FBP1 promotes $\mathrm{c}-\mathrm{Myc}$ degradation independent of its enzymatic activity. Furthermore, we constructed a mutant FBP1 nuclear export sequence (nes) linked to the C-terminus of FBP1 [15] (Fig. 5k). The overexpression of FBP1 nes had a similar impact as the wild-type FBP1 on c-Myc stability (Fig. $5 \mathrm{l}$ and m). These data suggest that FBP1 promotes c-Myc degradation primarily in the cytoplasm.

\section{FBP1 downregulates c-Myc through inhibition of the ERK1/2 pathway in PDAC}

It has previously been reported that FBP1 inhibits the IQGAP1-ERK interaction and decreases the phosphorylation of ERK1/2 in pancreatic cancer [17]. Firstly, we demonstrated that JQ1 could decrease ERK1/2 phosphorylation (p-ERK1/2) and this process was attenuated by knockdown of FBP1 in PANC-1 cells (Fig. 6a), indicating that BET inhibitors regulate ERK activation through FBP1. Furthermore, 90\% of PDAC patients were found to harbor Ras mutants [28]. Ras signaling and effector extracellular signal-regulated kinase (ERK) activation lead to the stabilization of c-Myc by attenuating its ubiquitin-mediated protein degradation $[29,30]$. We sought to determine whether FBP1 destabilize c-Myc in an IQGAP1-ERK pathway-dependent manner. The knockdown of IQGAP1 not only decreased the phosphorylation of ERK and c-Myc protein levels but also blocked the effect of FBP1 on the downregulation of c-Myc in PANC-1 cells (Fig. 6b). Moreover, we treated PANC-1 cells with a MEK inhibitor (trametinib) to block ERK phosphorylation (Fig. 6c and d). In agreement with previous findings, the MEK inhibitor 


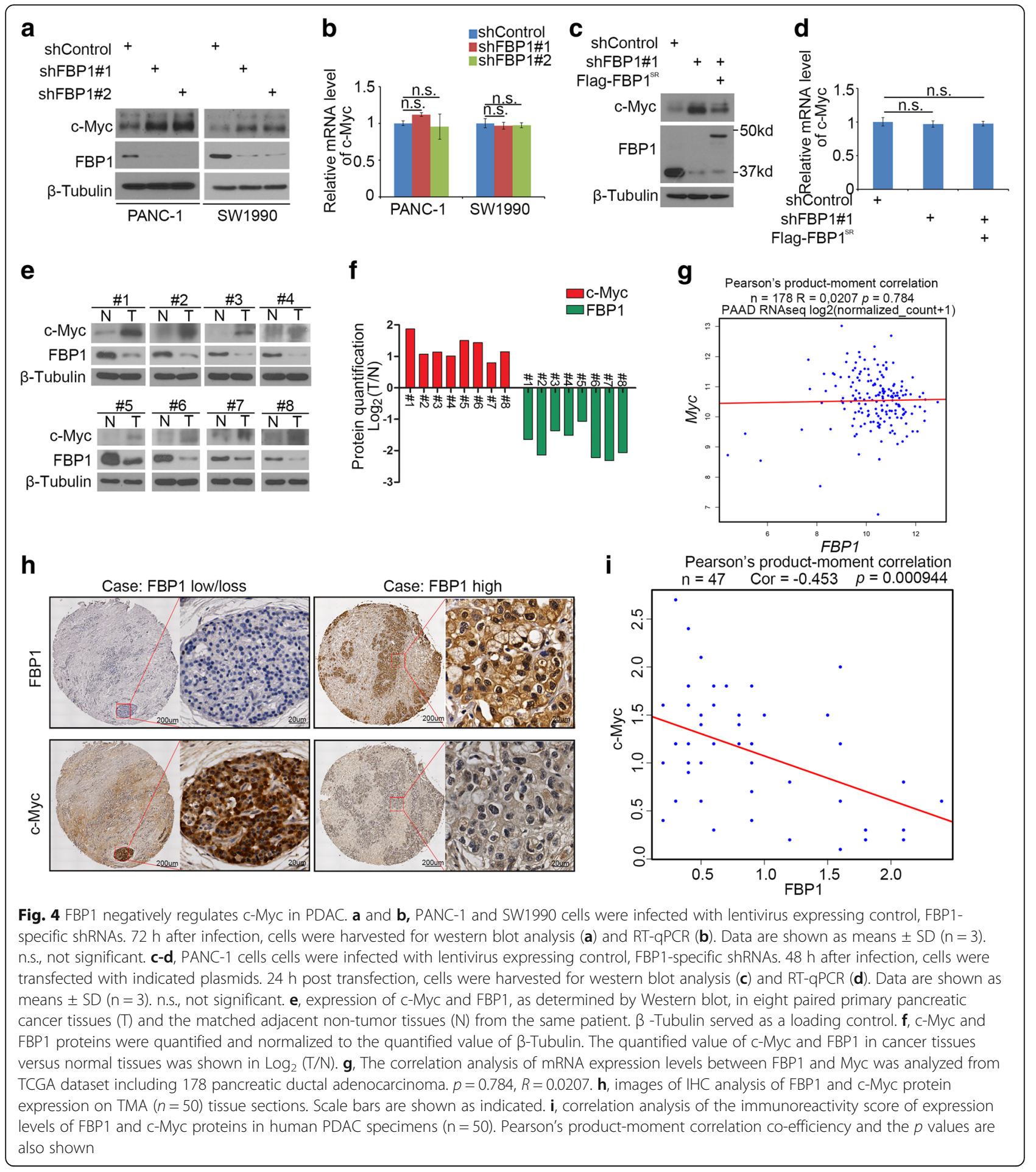

decreased c-Myc levels and ERK phosphorylation [31]. In addition, we found that the FBP1 knockdown (Fig. 6c) or the ectopic expression of FBP1 (Fig. 6d and e) failed to affect the expression of c-Myc in ERK-inactivated cells. Thus, our data suggest that FBP1 promotes c-Myc degradation through the inhibition of the ERK pathway in pancreatic cancer cells, and the
BET inhibitor downregulates c-Myc partially via stabilizing FBP1 in pancreatic cancer cells.

Previous studies have shown that FBP1 is downregulated in many types of solid tumors and that the restoration of FBP1 inhibits tumor cell proliferation [17]. Since our data showed that FBP1 promoted c-Myc degradation, we want to investigate the role of c-Myc on 


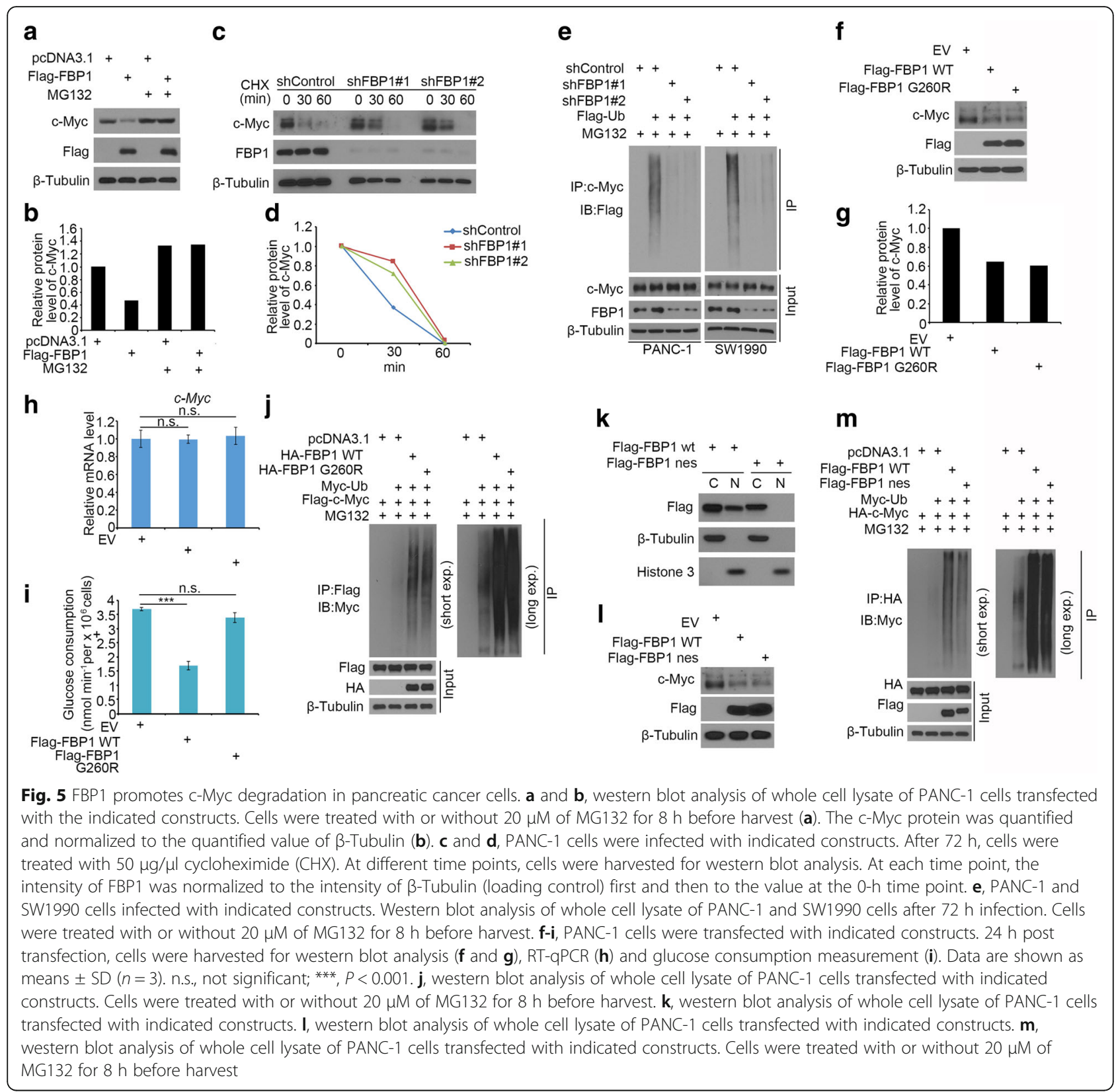

FBP1-mediated tumor suppression. We infected PANC-1 cells with the control, FBP1- and/or c-Myc-specific shRNA and harvested the lentivirus-infected cells for RT-qPCR, MTS assay, colony formation and animal studies. The knockdown of FBP1 increased the mRNA expression of c-Myc downstream target genes (HSPA4 and ENO1) [32], and this process was impeded by the knockdown of c-Myc in PANC-1 cells (Fig. 6f). MTS and colony formation assays showed that the knockdown of FBP1 increased cell growth, while the co-knockdown of c-Myc attenuated FBP1 knockdown-induced cell proliferation (Fig. 6g-h). Furthermore, the knockdown of FBP1 alone promoted the tumor growth in mice, but the knockdown of c-Myc alone inhibited this growth (Fig. 6j-6l). The co-knockdown of FBP1 and c-Myc blocked FBP1 knockdown-induced tumor growth in mice (Fig. 6k-6l). These data suggest that FBP1 decreases pancreatic cancer cell growth via inhibition of ERK-c-Myc pathway.

\section{Discussion}

FBP1 is a rate-limiting enzyme in gluconeogenesis that antagonizes glycolysis by converting fructose- 1 , 6-bisphosphate to fructose-6-phosphate and inorganic phosphate [27]. It is considered a tumor suppressor gene due to its role in the inhibition of aerobic glycolysis and subsequently impeding cancer cell proliferation [15]. 
a

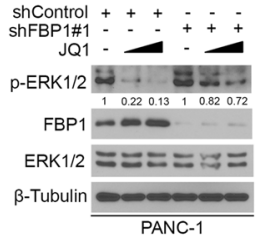

C $\underset{\text { Trametinib }}{\mathrm{DMSO}}+++$

$\mathrm{Flag}-\mathrm{FBP} P^{+}{ }^{+}+{ }^{+}+$

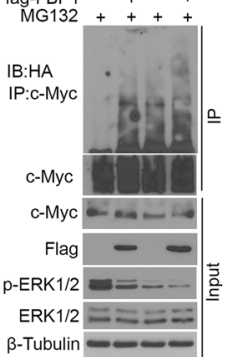

h

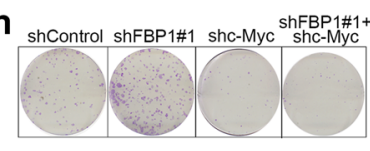

i

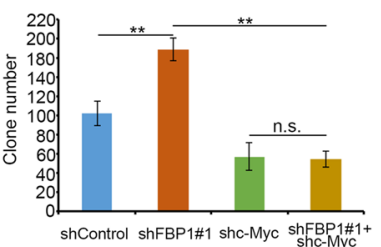

I

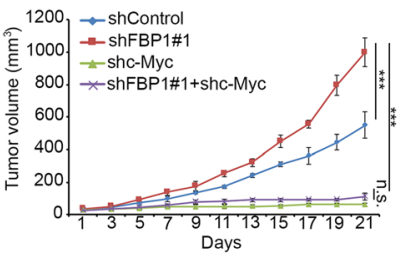

b

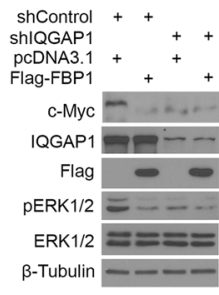

f

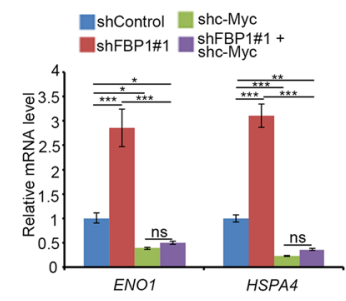

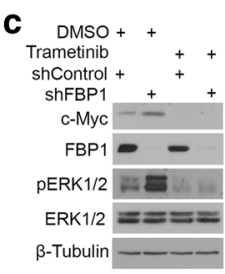

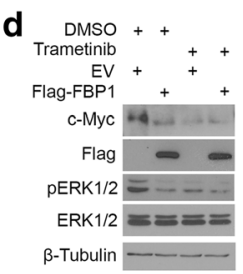

$\mathbf{g}$

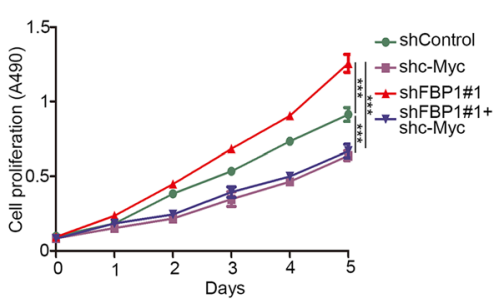

$\mathbf{k}$ j

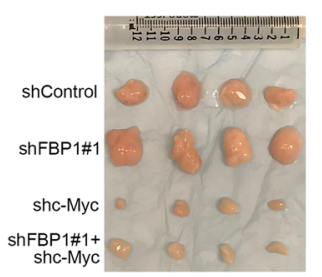

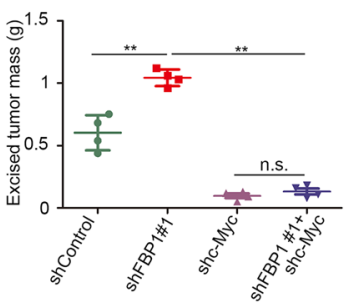

m

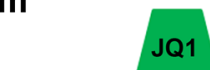

TRIM28

Fig. 6 FBP1 downregulates c-Myc through inhibition of the ERK1/2 pathway in PDAC. a, PANC-1 cells were infected with control or FBP1-specific shRNAs. After $24 \mathrm{~h}$, cells were treated with or without $2 \mu \mathrm{M}$ of JQ1 for another $24 \mathrm{~h}$. Cells were harvested for western blot analysis. b, PANC-1 cells were infected with lentivirus expressing control, IQGAP1-specific shRNAs. After $48 \mathrm{~h}$ infection, cells were transfected with indicated constructs and harvested for western blot analysis $24 \mathrm{~h}$ post transfection. Cells were treated with or without $20 \mu \mathrm{M}$ of MG132 for $8 \mathrm{~h}$ before being harvested. c, PANC-1 cells were infected with lentivirus expressing control, IQGAP1-specific shRNAs. After $48 \mathrm{~h}$ infection, cells were treated with $10 \mu \mathrm{M}$ of Trametinib for $24 \mathrm{~h}$ and harvested for western blot analysis. d, PANC-1 cells were transfected with indicated plasmids for $24 \mathrm{~h}$ and treated with $10 \mu \mathrm{M}$ of Trametinib for another $24 \mathrm{~h}$, and harvested for western blot analysis. e, PANC-1 cells were transfected with indicared plasmids. $24 \mathrm{~h}$ post transfection, cells were treated with $10 \mu \mathrm{M}$ of Trametinib for $24 \mathrm{~h}$. Cells were harvested for western blot analysis. Cells were treated with $20 \mu \mathrm{M}$ of MG132 for $8 \mathrm{~h}$ before harvest. f-i, PANC-1 cells were infected with lentivirus expressing control, or c-Myc and/or FBP1specific shRNAs. After $48 \mathrm{~h}$ infection and puromycin selection, cells were harvested for RT-qPCR analysis (f), MTS assay (g) and colony formation assay ( $h$ and $\mathbf{i}$ ) after $48 \mathrm{~h}$ infection. For rt-qPCR, all data show mean values \pm SD (error bar) $(n=3)$; for MTS assay, data are shown as means \pm SD $(n=6)$; for colony formation assay, data are shown as means \pm SD $(n=3)$. n.S., not significant; ${ }^{*} P<0.05 ; *^{*} P<0.01 ;{ }^{* *} P<0.001$. j-l, After $72 \mathrm{~h}$ infection, cells were injected into subcutaneous layer of the right flank of mice. The growth of tumor was measured every other day for 21 days (I), and tumors were harvested and measured the weight of tumor $(\mathbf{k})$. Data present means \pm SD $(n=4)$. n.s., not significant; ${ }^{* *} P<0.01$; ${ }^{* * *} P<$ 0.001. ( $\mathbf{m})$ A hypothetical model depicting JQ1 disrupts the interaction between FBP1 and TRIM28 and stabilizes FBP1.Next, FBP1 promoted c-Myc degradation through inhibiting the activation of ERK1/2. FBP1 serves as a therapeutic niche for patient-tailored therapies

Indeed, the downregulation of FBP1 leads to tumor progression and poor prognosis in pancreatic cancer [17], and this is also the case in various types of tumor $[27$,
33, 34]. Therefore, an up-regulation of FBP1 in pancreatic cancer might provide an ideal strategy to inhibit the pancreatic cancer cell progression. The specific 
mechanism underlying FBP1 downregulation is associated with promoter methylation and copy-number loss, leading to decreased FBP1 mRNA expression [22, 35] or post-transcriptional modification of FBP1 protein mediated by MAGE-TRIM28 complex, which results in FBP1 degradation in cancer cells [27]. Our findings in this study suggest that JQ1 increases FBP1 stability through disrupting the interaction between FBP1 and TRIM28, which suggest JQ1 might be an ideal small molecular to increase the FBP1 expression and repress pancreatic cancer.

A number of bromodomain inhibitors are currently being tested in several clinical trials [36], making those potentially promising drugs for the treatment of pancreatic cancer. BET inhibitor resistance often emerges in various cancer types [3, 37, 38]. Many factors, such as increasing the BRD4 protein level [24, 39, 40], were proved to lead drug resistance to BET inhibitors. Our data identified that FBP1 was a novel factor influencing the sensitivity of BET inhibitors. Due to c-Myc mediating the resistance of pancreatic cancer cells to the BET bromodomain inhibitor JQ1 $[9,10]$, our data also show that FBP1 decreases the expression of c-Myc in pancreatic cancer, which provides a reasonable mechanism to explain how FBP1 sensitized BET inhibitors treatment in pancreatic cancer.

Approximately $90 \%$ of PDAC patients possess Ras mutations [28]. It has been reported that Ras signaling and effector extracellular signal-regulated kinase (ERK) activation lead to the stabilization of c-Myc by attenuating its ubiquitin-mediated protein degradation [29, 30]. It is worth noting that FBP1 could bind to the WW domain of IQGAP1 and block IQGAP1-dependent ERK1/2 phosphorylation in pancreatic cancer [17]. In this study, we demonstrate that FBP1 promotes c-Myc degradation by inhibiting the MAPK pathway in pancreatic cancer cells. Intriguingly, it has been well documented that BRD4 directly regulates c-Myc expression at the transcriptional level [41]. In addition, it is reported that JQ1 decreases the mRNA level of c-Myc [42]. While, combined with our previous findings, the BET inhibitor increases FBP1 protein level which promoted c-Myc degradation in pancreatic cancer cells. Therefore, our data suggest that BET inhibitors regulate c-Myc expression not only in the transcription level but also in the post-transcription level.

\section{Conclusions}

FBP1 is responsible for the sensitivity of treatment with BET inhibitors in pancreatic cancer. JQ1 increases FBP1 protein level by disruption the interaction between TRIM28 and FBP1 (Fig. $3 j$ and $6 \mathrm{~m}$ ). Moreover, we show that FBP1 promotes c-Myc degradation through inhibition of the MAPK pathway (Fig. $6 \mathrm{~m}$ ). Together, our data suggest that FBP1 modulates the sensitivity of BET inhibitors by decreasing the expression of c-Myc in pancreatic cancer. These findings highlight FBP1 as a therapeutic niche for patient-tailored therapies.

\section{Additional file}

Additional file 1: Figure S1. BRD2, BRD3 or BRD4 make no effect on the expression of FBP1. Table S2. Sequences for shRNAs. (ZIP $272 \mathrm{~kb}$ )

\section{Abbreviations}

BET: Bromodomain and extraterminal domain; CHX: cycloheximide; co-IP: Coimmunoprecipitation; FBP1: Fructose-1, 6-biphosphatase; GST: Glutathione Stransferase; PDAC: Pancreatic ductal adenocarcinoma

\section{Acknowledgements}

We are grateful to Dr. Haojie Huang (Mayo Clinic) for providing FBP1 constructs.

\section{Funding}

This work was supported in part by grants from the Chinese National Natural Science Foundation Grant No. 81702374.

\section{Availability of data and materials}

Please contact author for data requests.

\section{Authors' contributions}

BW and PF performed the experiments and wrote the paper, XJ, HW and HW wrote the paper and convinces the data. All authors read and approved the final manuscript.

\section{Ethics approval and consent to participate}

The study was conducted in accordance with the Declaration of Helsinki principles. It was approved by the Animal Use and Care Committees at Tongji medical college, Huazhong University of Science and Technology.

\section{Consent for publication}

Not applicable.

\section{Competing interests}

The authors declare that they have no competing interests.

\section{Publisher's Note}

Springer Nature remains neutral with regard to jurisdictional claims in published maps and institutional affiliations.

\section{Author details}

${ }^{1}$ Department of Pancreatic Surgery, Union Hospital, Tongji Medical College, Huazhong University of Science and Technology, Wuhan 430022, China. ${ }^{2}$ Department of Digestive Surgical Oncology, Union Hospital, Tongji Medical College, Huazhong University of Science and Technology, Wuhan 430022, China. ${ }^{3}$ Operating Room, Union Hospital, Tongji Medical College, Huazhong University of Science and Technology, Wuhan 430022, China.

Received: 5 July 2018 Accepted: 21 August 2018

Published online: 10 September 2018

\section{References}

1. Hidalgo M. Pancreatic cancer. N Engl J Med. 2010;362:1605-17.

2. Von Hoff DD, Ervin T, Arena FP, Chiorean EG, Infante J, Moore M, Seay T, Tjulandin SA, Ma WW, Saleh MN, et al. Increased survival in pancreatic cancer with nab-paclitaxel plus gemcitabine. N Engl J Med. 2013;369:1691703.

3. Rathert P, Roth M, Neumann T, Muerdter F, Roe JS, Muhar M, Deswal S, Cerny-Reiterer S, Peter B, Jude J, et al. Transcriptional plasticity promotes primary and acquired resistance to BET inhibition. Nature. 2015;525:543-7.

4. Mazur PK, Herner A, Mello SS, Wirth M, Hausmann S, Sanchez-Rivera FJ, Lofgren SM, Kuschma T, Hahn SA, Vangala D, et al. Combined inhibition of 
BET family proteins and histone deacetylases as a potential epigeneticsbased therapy for pancreatic ductal adenocarcinoma. Nat Med. 2015;21: 1163-71.

5. Lenhart R, Kirov S, Desilva H, Cao J, Lei M, Johnston K, Peterson R, Schweizer $L$, Purandare A, Ross-Macdonald $P$, et al. Sensitivity of small cell lung Cancer to BET inhibition is mediated by regulation of ASCL1 gene expression. Mol Cancer Ther. 2015;14:2167-74.

6. Blee AM, Liu S, Wang L, Huang H. BET bromodomain-mediated interaction between ERG and BRD4 promotes prostate cancer cell invasion. Oncotarget. 2016;7:38319-32

7. Sahai V, Kumar K, Knab LM, Chow CR, Raza SS, Bentrem DJ, Ebine K, Munshi HG. BET bromodomain inhibitors block growth of pancreatic cancer cells in three-dimensional collagen. Mol Cancer Ther. 2014;13:1907-17.

8. Andricovich J, Perkail S, Kai Y, Casasanta N, Peng W, Tzatsos A. Loss of KDM6A activates super-enhancers to induce gender-specific squamous-like pancreatic Cancer and confers sensitivity to BET inhibitors. Cancer Cell. 2018;33:512-26. e518

9. Bian B, Bigonnet M, Gayet O, Loncle C, Maignan A, Gilabert M, Moutardier V, Garcia S, Turrini O, Delpero JR, et al. Gene expression profiling of patientderived pancreatic cancer xenografts predicts sensitivity to the BET bromodomain inhibitor JQ1: implications for individualized medicine efforts. EMBO Mol Med. 2017;9:482-97.

10. Kumar K, Raza SS, Knab LM, Chow CR, Kwok B, Bentrem DJ, Popovic R, Ebine K, Licht JD, Munshi HG. GLI2-dependent C-MYC upregulation mediates resistance of pancreatic cancer cells to the BET bromodomain inhibitor JQ1. Sci Rep. 2015;5:9489.

11. Wirth M, Mahboobi S, Kramer OH, Schneider G. Concepts to target MYC in pancreatic Cancer. Mol Cancer Ther. 2016;15:1792-8.

12. Kortlever RM, Sodir NM, Wilson CH, Burkhart DL, Pellegrinet $L$, Brown Swigart L, Littlewood TD, Evan GI. Myc cooperates with Ras by programming inflammation and immune suppression. Cell. 2017;171:130115. e1314

13. Xin B, Yamamoto M, Fujii K, Ooshio T, Chen X, Okada Y, Watanabe K, Miyokawa N, Furukawa H, Nishikawa Y. Critical role of Myc activation in mouse hepatocarcinogenesis induced by the activation of AKT and RAS pathways. Oncogene. 2017;36:5087-97.

14. Jiang SH, Li J, Dong FY, Yang JY, Liu DJ, Yang XM, Wang YH, Yang MW, Fu $X \mathrm{~L}$, Zhang $X X$, et al. Increased serotonin signaling contributes to the Warburg effect in pancreatic tumor cells under metabolic stress and promotes growth of pancreatic tumors in mice. Gastroenterology. 2017;153: 277-91. e219

15. Li B, Qiu B, Lee DS, Walton ZE, Ochocki JD, Mathew LK, Mancuso A, Gade TP, Keith B, Nissim I, Simon MC. Fructose-1,6-bisphosphatase opposes renal carcinoma progression. Nature. 2014;513:251-5.

16. Shi S, Ji S, Qin Y, Xu J, Zhang B, Xu W, Liu J, Long J, Liu C, Liu L, et al. Metabolic tumor burden is associated with major oncogenomic alterations and serum tumor markers in patients with resected pancreatic cancer. Cancer Lett. 2015;360:227-33.

17. Jin X, Pan Y, Wang L, Ma T, Zhang L, Tang AH, Billadeau DD, Wu H, Huang $H$. Fructose-1,6-bisphosphatase inhibits ERK activation and bypasses gemcitabine resistance in pancreatic Cancer by blocking IQGAP1-MAPK interaction. Cancer Res. 2017;77:4328-41.

18. Jin X, Yang C, Fan P, Xiao J, Zhang W, Zhan S, Liu T, Wang D, Wu H. CDK5/ FBW7-dependent ubiquitination and degradation of EZH2 inhibits pancreatic cancer cell migration and invasion. J Biol Chem. 2017;292:6269-80

19. Jin X, Yan Y, Wang D, Ding D, Ma T, Ye Z, Jimenez R, Wang L, Wu H, Huang $H$. DUB3 promotes BET inhibitor resistance and Cancer progression by deubiquitinating BRD4. Mol Cell. 2018;

20. Yang J, Jin X, Yan Y, Shao Y, Pan Y, Roberts LR, Zhang J, Huang H, Jiang J. Inhibiting histone deacetylases suppresses glucose metabolism and hepatocellular carcinoma growth by restoring FBP1 expression. Sci Rep. 2017;7:43864.

21. Yu J, Li J, Chen Y, Cao W, Lu Y, Yang J, Xing E. Snail enhances glycolysis in the epithelial-mesenchymal transition process by targeting FBP1 in gastric Cancer. Cell Physiol Biochem. 2017;43:31-8.

22. Dong C, Yuan T, Wu Y, Wang Y, Fan TW, Miriyala S, Lin Y, Yao J, Shi J, Kang T, et al. Loss of FBP1 by snail-mediated repression provides metabolic advantages in basal-like breast cancer. Cancer Cell. 2013;23:316-31.

23. Filippakopoulos P, Qi J, Picaud S, Shen Y, Smith WB, Fedorov O, Morse EM, Keates T, Hickman TT, Felletar I, et al. Selective inhibition of BET bromodomains. Nature. 2010;468:1067-73.
24. Zhang P, Wang D, Zhao Y, Ren S, Gao K, Ye Z, Wang S, Pan CW, Zhu Y, Yan $Y$, et al. Intrinsic BET inhibitor resistance in SPOP-mutated prostate cancer is mediated by BET protein stabilization and AKT-mTORC1 activation. Nat Med. 2017:23:1055-62.

25. Yan Y, An J, Yang Y, Wu D, Bai Y, Cao W, Ma L, Chen J, Yu Z, He Y, et al. Dual inhibition of AKT-mTOR and AR signaling by targeting HDAC3 in PTEN- or SPOP-mutated prostate cancer. EMBO Mol Med. 2018;10

26. Kimura $Y$, Tanaka K. Regulatory mechanisms involved in the control of ubiquitin homeostasis. J Biochem. 2010;147:793-8.

27. Jin X, Pan Y, Wang L, Zhang L, Ravichandran R, Potts PR, Jiang J, Wu H, Huang H. MAGE-TRIM28 complex promotes the Warburg effect and hepatocellular carcinoma progression by targeting FBP1 for degradation. Oncogenesis. 2017;6:e312.

28. Bos JL. Ras oncogenes in human cancer: a review. Cancer Res. 1989;49: 4682-9.

29. Tsai WB, Aiba I, Long Y, Lin HK, Feun L, Savaraj N, Kuo MT. Activation of Ras/ PI3K/ERK pathway induces c-Myc stabilization to upregulate argininosuccinate synthetase, leading to arginine deiminase resistance in melanoma cells. Cancer Res. 2012;72:2622-33.

30. Hayes TK, Neel NF, Hu C, Gautam P, Chenard M, Long B, Aziz M, Kassner M, Bryant KL, Pierobon M, et al. Long-term ERK inhibition in KRAS-mutant pancreatic Cancer is associated with MYC degradation and senescence-like growth suppression. Cancer Cell. 2016;29:75-89.

31. Togel $L$, Nightingale $R$, Chueh $A C$, Jayachandran $A$, Tran $H$, Phesse $T$, Wu $R$, Sieber OM, Arango D, Dhillon AS, et al. Dual targeting of Bromodomain and Extraterminal domain proteins, and WNT or MAPK signaling, inhibits C-MYC expression and proliferation of colorectal Cancer cells. Mol Cancer Ther. 2016;15:1217-26.

32. Zeller KI, Jegga AG, Aronow BJ, O'Donnell KA, Dang CV. An integrated database of genes responsive to the Myc oncogenic transcription factor: identification of direct genomic targets. Genome Biol. 2003;4:R69.

33. Li J, Wang Y, Li QG, Xue JJ, Wang Z, Yuan X, Tong JD, Xu LC. Downregulation of FBP1 promotes tumor metastasis and indicates poor prognosis in gastric Cancer via regulating epithelial-mesenchymal transition. PLoS One. 2016;11:e0167857.

34. Cong J, Wang X, Zheng X, Wang D, Fu B, Sun R, Tian Z, Wei H. Dysfunction of natural killer cells by FBP1-induced inhibition of glycolysis during lung Cancer progression. Cell Metab. 2018;28:243-55. e245

35. Pan D, Mao C, Wang YX. Suppression of gluconeogenic gene expression by LSD1-mediated histone demethylation. PLoS One. 2013;8:e66294.

36. Andrieu G, Belkina AC, Denis GV. Clinical trials for BET inhibitors run ahead of the science. Drug Discov Today Technol. 2016;19:45-50.

37. Fong $C Y$, Gilan $O$, Lam EY, Rubin AF, Ftouni S, Tyler D, Stanley K, Sinha D, Yeh $\mathrm{P}$, Morison J, et al. BET inhibitor resistance emerges from leukaemia stem cells. Nature. 2015;525:538-42

38. Shu S, Lin CY, He HH, Witwicki RM, Tabassum DP, Roberts JM, Janiszewska M, Huh SJ, Liang Y, Ryan J, et al. Response and resistance to BET bromodomain inhibitors in triple-negative breast cancer. Nature. 2016;529: 413-7.

39. Janouskova H, El Tekle G, Bellini E, Udeshi ND, Rinaldi A, Ulbricht A, Bernasocchi T, Civenni G, Losa M, Svinkina T, et al. Opposing effects of cancer-type-specific SPOP mutants on BET protein degradation and sensitivity to BET inhibitors. Nat Med. 2017:23:1046-54.

40. Dai X, Gan W, Li X, Wang S, Zhang W, Huang L, Liu S, Zhong Q, Guo J, Zhang J, et al. Prostate cancer-associated SPOP mutations confer resistance to BET inhibitors through stabilization of BRD4. Nat Med. 2017;23:1063-71.

41. Bandukwala HS, Gagnon J, Togher S, Greenbaum JA, Lamperti ED, Parr NJ, Molesworth AM, Smithers N, Lee K, Witherington J, et al. Selective inhibition of CD4+ T-cell cytokine production and autoimmunity by BET protein and c-Myc inhibitors. Proc Natl Acad Sci U S A. 2012;109:14532-7.

42. Ott CJ, Kopp N, Bird L, Paranal RM, Qi J, Bowman T, Rodig SJ, Kung AL, Bradner JE, Weinstock DM. BET bromodomain inhibition targets both c-Myc and IL7R in high-risk acute lymphoblastic leukemia. Blood. 2012:120:2843-52 